\title{
MULTIPLE TRAFFIC JAMS IN FULL VELOCITY DIFFERENCE MODEL WITH REACTION-TIME DELAY
}

\author{
$\mathrm{Xu}, \mathrm{R} . \mathrm{G}$. \\ School of Aerospace Engineering and Applied Mechanics, Tongji University, Shanghai 200092, China \\ E-Mail: xurong.gai@163.com
}

\begin{abstract}
A full velocity difference model for traffic flow, including driver's reaction time delay is considered. The uniform flow and traffic jams are interpreted through stability and bifurcation analysis. Specifically, the uniform flow is represented by the equilibrium of the model. Linear stability reveals that when the equilibrium loses its stability, local bifurcation turn up through Hopf bifurcations. To analyse the behaviour of the model after bifurcating, numerical continuation techniques are employed. Branches of oscillating solutions and the corresponding stabilities are obtained. It is shown that bifurcating oscillations can coexist and correspond to different traffic patterns. To visualize the spatial patterns, numerical simulations are performed, which are presented by velocity time histories and spatio-temporal diagrams. Analysing the characteristic features, these oscillating solutions are classified into three types, and further correspond to three types of traffic jams: almost traffic jams, width-equal traffic jam and width-alternated traffic jam. The obtained results provide an explanation of how multiple jams induced by driver's reaction time delay occur.

(Received, processed and accepted by the Chinese Representative Office.)
\end{abstract}

Key Words: Full Velocity Difference Model, Reaction-Time Delay, Stability, Bifurcation, Numerical Continuation, Traffic Patterns

\section{INTRODUCTION}

In recent years, traffic flow problems have received considerable interest from various disciplines [1]. One of the reasons is that traffic jams are becoming more and more serious problems. However, although part of our daily lives, traffic dynamics and especially, traffic jams, have not been well understood. To study the traffic dynamics and to understand the mechanism of traffic jams, modelling of the traffic flow is the first step. In continuum models, instead of considering individual vehicles, continuous density distribution and velocity distribution (as functions of space and time) are used to describe the traffic flow, where partial differential equations (PDEs) are employed to describe the traffic dynamics [2]. In cellular automata models, space, time and velocity are considered to be discrete, and updated rules are used to describe the time development of traffic flow [3]. In car-following models, discrete entities move in continuous space and time, and the vehicles' motions are described by ordinary differential equations (ODEs) [4] or by delay differential equations (DDEs) if time delay is introduced [5]. The optimal velocity (OV) model [4] is the most widely used car-following model. Generally, the quality of these developed models is evaluated by fitting the models to empirical data [6]. The data-fitting method easily leads to research capturing. However, some essential characteristics of traffic dynamics, e.g., the coexistence of multiple stable traffic flow, cannot be fully revealed through such data-fitting method. Hence, to comprehensively understand and classify the characteristic features of traffic flow, especially, the 'hidden' unstable motion, general models with varying traffic flow parameters need to be studied [7].

Among the previous research, bifurcation theory is a useful and effective way to study the dynamical behaviour of the traffic flow models. Igarashi et al. [8] first considered the bifurcation phenomena in a car-following model and pointed out that the subcritical Hopf bifurcation was the dynamical origin of the evolution between uniform flow and congested 
traffic flow. Hopf bifurcation was investigated through numerical continuation techniques [9] by Gasser et al. [10]. Notice that during driving, due to human physiological factors, drivers require a finite period of time to process stimuli from the preceding vehicle, which inherently introduces the drivers' reaction time delay into the car-following models. In 1936, driver's reaction time delay was first recognized in traffic dynamics [11]. However, it was first introduced into the OV model in 1998, where drivers were supposed to react to their current velocity and headway with the same time delay [12]. Davis [13] proposed a modified time-delayed OV model, which assumed that drivers could recognize their speed without time delay, but need some time to react to their headway. This model was used by Yu et al. [14] to investigate the density waves, and by Orosz et al. [15] to perform global bifurcation analysis through numerical continuation techniques [16]. A new car-following model considering the effects of two different time delays was analysed in [17]. In the above OV models, drivers' reaction does not depend on the velocity difference of the vehicles. However, drivers' reaction should depend on not only the changes in headway, but more importantly, the velocity difference between vehicles [18]. Shamoto [19] pointed out that the acceleration of a vehicle had a positive correlation with the velocity difference.

The presented research is motivated by two aspects. On one hand, an appropriate car-following model should include the headway, current own velocity and velocity difference. Moreover, drivers' reaction time delay with respect to these factors should be contained, because a recent study [20] has pointed out that drivers' reaction time may lead to unstable traffic flow. On the other hand, exploring all the possible dynamic behaviour of the model with respect to varying model parameters, especially the driver's reaction time delay, is of great significance to the comprehensive understanding of the mechanism of traffic jams. To do so, in this paper, a full velocity difference model with drivers' reaction time delay is built. Through linear analysis, uniform flow stability is studied and the stability boundary of the uniform flow is obtained. Then, based on numerical continuation techniques, the bifurcation behaviour of the model when the uniform flow loses stability is investigated with respect to drivers' reaction time delay. It is found that multiple oscillating solutions with distinct characteristics can coexist. Through numerical simulations, these oscillating solutions and their corresponding traffic patterns are studied and classified into three types.

\section{TIME-DELAYED FULL VELOCITY DIFFERENCE MODEL}

In this section, a time-delayed full velocity difference model is built. Suppose that $n$ identical vehicles driven by $n$ identical drivers are placed on a circular road, where overtaking and lane-changing effects are ignored. Consider the situation that the $i^{\text {th }}$ vehicle follows the $i+1^{\text {st }}$ vehicle, and the $n^{\text {th }}$ vehicle follows the first one. Denote the position of each vehicle by $x_{i}$ $(i=1,2, \ldots, n)$, the velocity by $v_{i}$. Hence, the relative displacements (called headways) $h_{i}(t)$ can be expressed as $h_{i}(t)=x_{i+1}(t)-x_{i}(t)$. Taking the time derivative of $h_{i}(t)$ yields the relative velocity of two adjacent vehicles (called velocities difference):

$$
\dot{h}_{i}(t)=v_{i+1}(t)-v_{i}(t), i=1,2, \ldots, n-1
$$

Here the dot denotes the derivation with respect to time. Assume that the length of the circular road is $L$ and the length of each vehicle can be neglected. Since the $n^{\text {th }}$ car follows the first car, it is reasonable to get $h_{n}=L-\sum_{i=1}^{n-1} h_{i}$, such that:

$$
\dot{h}_{n}(t)=v_{1}(t)-v_{n}(t)
$$

The original OV model was developed by Bando et al. [4], i.e.:

$$
\dot{v}_{i}(t)=\alpha\left[V\left(h_{i}(t)\right)-v_{i}(t)\right]
$$


Introducing the velocity difference into the eq. (3) yields the full-velocity deference model [21], i.e.:

$$
\dot{v}_{i}(t)=\alpha\left[V\left(h_{i}(t)\right)-v_{i}(t)\right]+\beta \dot{h}_{i}(t)
$$

In eqs. (3) and (4), the parameters $\alpha>0$ and $\beta>0$ are the sensitivity, and $V\left(h_{i}\right)$ is known as the optimal velocity function. Models considering the time delay in reaction to the headway and to its own velocity are developed in [12] and [13], respectively.

The presented model specifies that the acceleration of each vehicle is a function of the three stimuli that the driver receives: the headways, the current velocities and the velocity difference. Besides, it is assumed that the drivers' reaction time delay with respect to all these three stimuli was not zero. Denote $\tau_{1}, \tau_{2}$ and $\tau_{3}$ as driver's reaction time delay with respect to the stimuli of headway, its own velocity and velocity difference, respectively. Thus the acceleration of the $i^{\text {th }}$ vehicle can be expressed as:

$$
\dot{v}_{i}(t)=\alpha\left[V\left(h_{i}\left(t-\tau_{1}\right)\right)-v_{i}\left(t-\tau_{2}\right)\right]+\beta \dot{h}_{i}\left(t-\tau_{3}\right)
$$

Owing to the non-zero time delay, such model is named as time-delayed full velocity difference model. Combining eqs. (1), (2) and (5) and using the human driver set-up in [7] $\left(\tau_{1}=\tau_{3}=\tau, \tau_{2}=0\right)$ yields our dynamic model expressed by delay differential equations:

$$
\left\{\begin{array}{l}
\dot{h}_{i}(t)=v_{\mathrm{i}+1}(t)-v_{\mathrm{i}}(t) \\
\dot{v}_{i}(t)=\alpha\left(V\left(h_{i}(t-\tau)\right)-v_{\mathrm{i}}(t)\right)+\beta\left(v_{\mathrm{i}+1}(t-\tau)-v_{\mathrm{i}}(t-\tau)\right) \\
\dot{v}_{n}(t)=\alpha\left(V\left(L-\sum_{i=1}^{n-1} h_{i}(t-\tau)\right)-v_{n}(t)\right)+\beta\left(v_{1}(t-\tau)-v_{n}(t-\tau)\right)
\end{array} \quad i=1, \ldots, n-1\right.
$$

Orosz presented a non-dimensionalized optimal velocity function [15], which will be used in this study, i.e.:

$$
V(h)= \begin{cases}0, & \text { if } h \in\left[0, h_{\text {stop }}\right] \\ v^{0} \frac{\left[\left(h-h_{\text {stop }}\right)\right]^{3}}{h_{\text {stop }}^{3}+\left(h-h_{\text {stop }}\right)^{3}}, & \text { if } h \in\left[h_{\text {stop }}, \infty\right)\end{cases}
$$

where $h_{\text {stop }}>0$ stands for a jam headway, and $v^{0}>0$ is the desired speed.

\section{LINEAR STABILITY ANALYSIS}

Uniform traffic flow is such a situation that equidistant cars move with the same time-independent velocity and zero velocity difference with respect to their nearby vehicle. Notice that uniform flow exists in the stable region of the linearized system. Hence, in what follows linear stability analysis will first be performed.

It is obvious that eq. (6) possesses uniform flow equilibrium:

$$
h_{i}(t)=h^{*}=\frac{L}{n}, v_{i}(t)=v^{*}=V\left(h^{*}\right), \dot{h}_{i}(t) \equiv 0, \quad i=1, \ldots, n
$$

In order to study the stability of this equilibrium, eq. (6) can be linearized as:

$$
\left\{\begin{array}{l}
\dot{h}_{n}(t)=v_{i+1}(t)-v_{i}(t) \\
\dot{h}_{i}(t)=-\alpha v_{i}(t)+\alpha V^{\prime}\left(h^{*}\right)\left(h_{i}(t-\tau)-h^{*}\right)+\beta\left(v_{i+1}(t-\tau)-v_{i}(t-\tau)\right) \quad i=1, \ldots, n-1 \\
\dot{v}_{n}(t)=-\alpha v_{n}(t)+\alpha V^{\prime}\left(h^{*}\right)\left(L-\sum_{i=1}^{n-1} h_{i}(t-\tau)-h^{*}\right)+\beta\left(v_{1}(t-\tau)-v_{n}(t-\tau)\right)
\end{array}\right.
$$


Using the trial solution $\mathrm{e}^{\lambda t}$ with $\lambda \in \mathrm{C}$, one obtains the characteristic equation in the form:

$$
(\alpha+\lambda) \prod_{k=1}^{n-1}\left(\lambda^{2}+\alpha \lambda+\mathrm{e}^{-\lambda \tau}\left(V^{\prime}\left(h^{*}\right) \alpha+\beta \lambda\right)-\mathrm{e}^{-\lambda \tau}\left(V^{\prime}\left(h^{*}\right) \alpha+\beta \lambda\right) \mathrm{e}^{\frac{2 \pi k}{n} i}\right)=0
$$

Substituting the critical eigenvalue $\lambda=i \omega, \omega>0$ into eq. (10), separating the real and imaginary parts and using some trigonometric identities, one obtains the Hopf stability curves in the $(\tau, \beta)$ plane:

$$
\left\{\begin{array}{l}
\tau=\frac{1}{\omega} \arccos \frac{\omega\left(\alpha\left(V^{\prime}\left(h^{*}\right)-\beta\right) \omega+\left(V^{\prime}\left(h^{*}\right) \alpha^{2}+\beta \omega^{2}\right) \cot \frac{k \pi}{n}\right)}{2\left(V^{\prime}\left(h^{*}\right)^{2} \alpha^{2}+\beta^{2} \omega^{2}\right)} \\
\beta=\frac{\sqrt{-4 V^{\prime}\left(h^{*}\right)^{2} \alpha^{2}+\omega^{2}\left(\alpha^{2}+\omega^{2}\right) \csc ^{2} \frac{k \pi}{n}}}{2 \omega}
\end{array}\right.
$$

Each curve of eq. (11) belongs to each spatial wave number $k$ of oscillations along the ring and is parameterized by the frequency $\omega$.

Noting that $k=1,2, \ldots, n-1$ is introduced by the $n^{\text {th }}$ of unity. For the following parameters:

$$
n=33, \alpha=0.9 \mathrm{~s}^{-1}, v^{0}=11 \mathrm{~m} / \mathrm{s}, h_{\text {stop }}=14 \mathrm{~m}, L=1122 \mathrm{~m}
$$

The Hopf stability curves corresponding to $k=1$ to $n-1$ are shown in Fig. 1 . The stability curves for $k \leq 11$ and $k \geq 12$ is denoted by blue and green curves, respectively. A solid arrow indicates the increase of the wave number. In the grey area of Fig. 1, the uniform flow is stable. Crossing any stability curve, a pair of complex-conjugate eigenvalues cross the imaginary axis, i.e., a Hopf bifurcation takes place. For small sensitivity $\beta(\beta<0.4)$, the curve corresponding to $k=1$ constitutes the stability boundary between the uniform flow and oscillations. With the increase of time delay, curves corresponding to $k>1$ result in further oscillation modes around the already unstable equilibrium. However, for large $\beta(\beta>0.4)$, $k=11$ and $k=12$ curves constitute the stability boundary, and oscillations corresponding to other values of $k$ show up alternately with the increase of time delay. Thus, one notices that even at the linear level the time delay can induce different spatial dynamics, which will be further analysed in sections 4 and 5 .

To demonstrate the complexity in the unstable uniform flow domain with varying time delay, further nonlinear analysis is necessary. We need to use the numerical continuation methods [16] to describe the global dynamics.

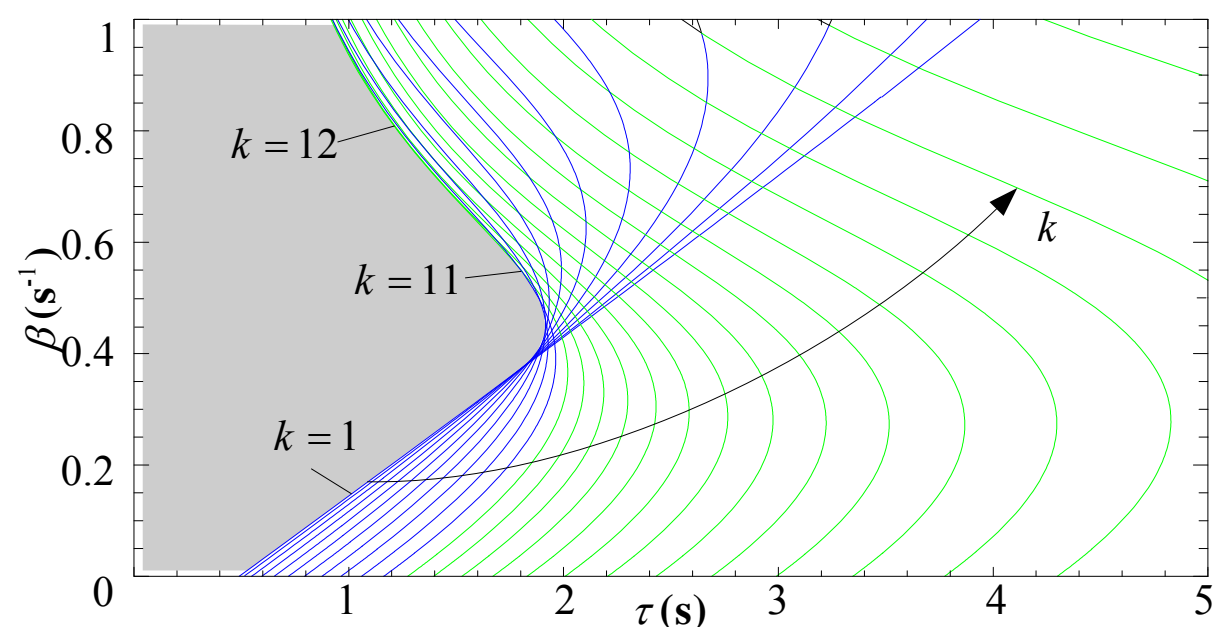

Figure 1: Linear stability diagrams for $n=33$ cars. 


\section{NUMERICAL BIFURCATION ANALYSIS}

In this section, numerical continuation techniques [16] are employed to demonstrate the complexity arisen at the nonlinear level when the drivers' reaction time delay varies. Bifurcation analysis of the time-delayed full velocity difference model is performed. The basic idea is to find a bifurcation with the change of time delay $\tau$ (the dynamics change qualitatively at a critical value) and then continue the bifurcation solution as $\tau$ is changed. To this end, the amplitude of the velocity oscillations is used as a function of reaction-time delay, which is defined as:

$$
v_{i, \text { amp }}=\max _{t} v_{i}(t)-\min _{t} v_{i}(t), \quad i=1, \ldots, n
$$

Since it is assumed that each vehicle and each driver possess the same characteristics, which lead to a $Z_{n}$ symmetry of the system, a result is:

$$
v_{i}(t)=v_{i+1}\left(t-\frac{T}{n}\right), h_{i}(t)=h_{i+1}\left(t-\frac{T}{n}\right)
$$

where $T$ is the period of the velocity oscillations. In other words, it is sufficient to plot the profile of the first car, and then the profiles for all other cars are simply shifted copies.

Since the studied system is described by infinite dimensional DDEs eq. (6), the software package DDE-BIFTOOL is adopted to find and continue both equilibrium and oscillating solutions of eq. (6), even if they are unstable. Considering the real traffic instance, one takes $\tau \in[0,1.5]$ as the continuation range [22]. With parameters in eq. (12) and $\beta=0.1$, numerical continuations are carried out. At the same time, to determine the stability of oscillating solutions, the Floquet multipliers are calculated. Fold bifurcation of oscillating solutions are detected, where two oscillating solutions merge and disappear. Neimark-Sacker bifurcation occurs where the oscillating solution changes its stability and quasiperiodic oscillation arises (the detail of which is not studied in this paper). A period-doubling bifurcation occurs where the oscillating solution changes its stability and even yields more complicate oscillation.
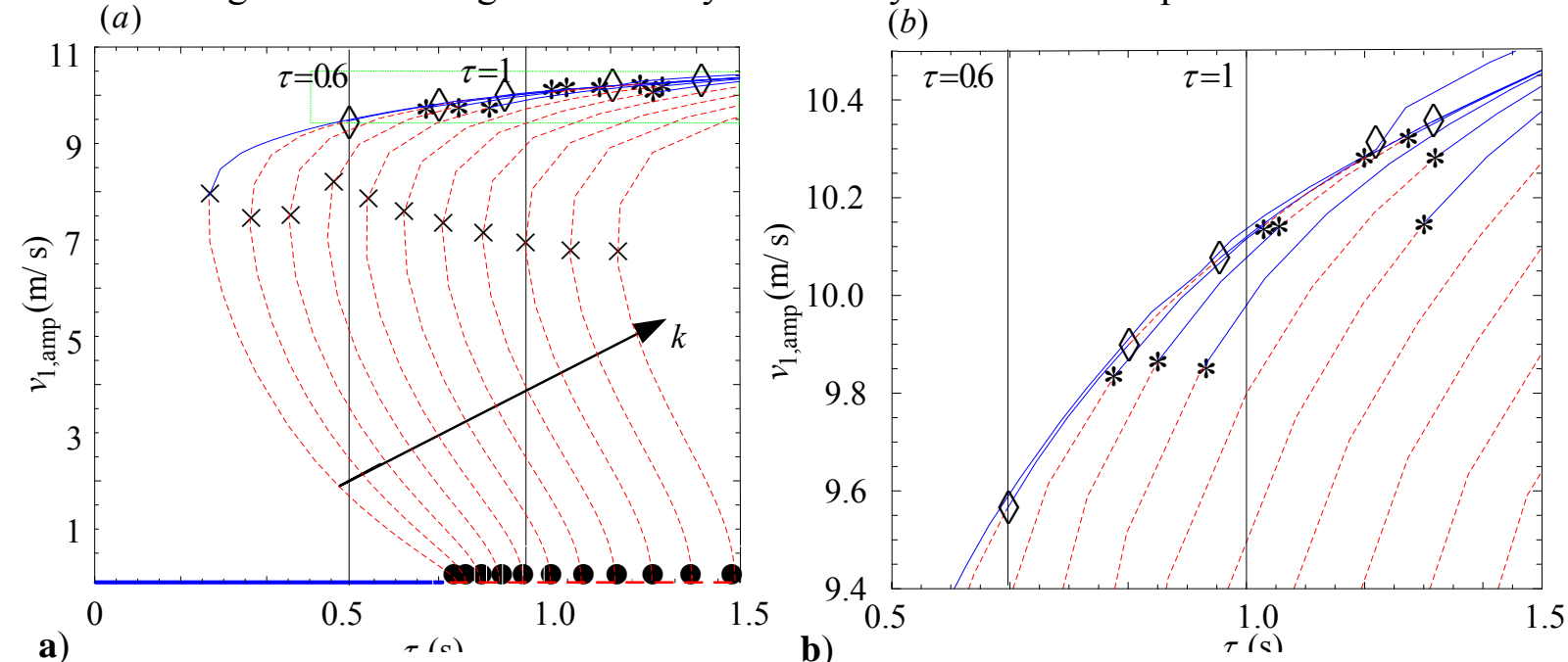

Figure 2: a) Solid and dashed curves indicate stable and unstable solutions, respectively;

b) Local zoom of the rectangle region in part a).

Fig. 2 shows the continuation results of the first car for different values of wave number $k$. The horizontal axis is the reaction-time delay $\tau$, and the vertical axis displays the amplitude of velocity oscillation of the first car for a different wave number. To clearly observe the bifurcations, the rectangular region in Fig. $2 \mathrm{a}$ is zoomed in Fig. 2 b. Stable and unstable solutions are denoted by solid curves and dashed curves respectively. Fold, Neimark-Sacker, and period-doubling bifurcations of the periodic oscillations are denoted by crosses $(\times)$, stars 
(*) and diamonds $(\diamond)$ respectively. The solid circles on the $v_{1, \text { amp }}=0$ line are the subcritical Hopf bifurcation points of uniform flow.

Note that the branch corresponding to $k=1$ belongs to the stability boundary of the uniform flow equilibrium, while the curves corresponding to $k>1$ result in further oscillation modes around the already unstable equilibrium. These branches of oscillations will undergo further bifurcations. By calculating the Floquet multipliers with the DDE-BIFTOOL, it is found that fold bifurcation $(\times)$ occurs on every branch $k$ where branches fold back. While period doubling bifurcations $(\diamond)$ only occur on the branches $k=2$ where the stability changes. Except the branches $k=1,2$, Neimark-Sacker bifurcations $(*)$ occur on all the other branches $(k=3,4,5, \ldots)$, where the stability also changes. One should notice that for $\beta=0.1, \tau_{C}=0.6$ (see the first vertical line shown in Fig. 2) is the first period-doubling bifurcations point corresponding to the wave number $k=2$, where two stable periodic oscillations corresponding to wave number $k=1$ and $k=2$ coexist.

Fig. 2 also shows that for any value of time delay that locates between $\left(\tau_{C}, 1.5\right)$, several stable and unstable periodic oscillations corresponding to different wave numbers $k$ can coexist. Here we say that the periodic oscillation is stable in this numerical continuation technique if the maximum Floquet multiplier is smaller than 1.09 , i.e. $\max |\mu| \leq 1.09$. It should be noted that to meet the requirements of precise numerical continuation, criteria $\max |\mu| \leq 1.09$ is employed, instead of the theoretical criteria max $|\mu| \leq 1$. To clearly describe the coexistence of stable and unstable periodic oscillations $\tau=1 \mathrm{~s}$ is taken as an example (see the second vertical line shown in Fig. 2). The results obtained from the DDE-BIFTOOL reveal that for $\tau=1$, the periodic oscillations are stable for the branches corresponding to $k=1, \ldots, 5$ but are unstable for the branches $k=6, \ldots, 9$.

To understand the evolution of each oscillation corresponding to a different wave number $k$, initial value simulations of the eq. (6) from suitable initial data are carried out $(\tau=1)$, results of which are shown in Fig. 3. It is found that for the wave numbers $k=1, \ldots, 5$ the velocity of the first car performs stable periodic oscillation. However, the oscillations related to wave numbers $k=6, \ldots, 9$ are unstable during the transient stage, as is predicted, but eventually evolve into stable oscillations, but of different types. Unstable periodic oscillations for $k=7$ and 8 become stable period-doubling oscillations, while unstable periodic oscillations for $k=6$ and 9 eventually become stable periodic oscillations. As a result, all the periodic oscillations corresponding to wave number $k=1, \ldots, 9$ finally evolve into stable forms, which can be further classified into two types: stable periodic oscillations for $k=1,2,3,4,5,6,9$ and stable period-doubling oscillation for $k=7,8$. In the next section, detailed characteristics of these oscillations and their corresponding traffic patterns will be discussed.

\section{MULTIPLE TRAFFIC JAMS WITH NUMERICAL SIMULATIONS}

To understand the traffic patterns corresponding to different wave numbers, in this section numerical simulations are performed for each case $(k=1, \ldots, 9)$ to visualize the final stable oscillation in time history diagrams (Fig. 3) and the resulting spatial patterns in spatiotemporal diagrams (Fig. 4).

In our model, we say that a traffic jam occurs if there is a region of the ring along which the cars remain stationary (velocity is less than $0.01 \mathrm{~m} / \mathrm{s}$ in our definition). We denote $T_{\text {jam }}$ as the cost time that the vehicles stay in the jams region.

We first look at the time history of the velocity of the first car for various wave numbers $k$ in Fig. 3. It is found that for $k=1,2,3,6,9$ there exist finite time intervals where the velocity $v_{1}$ is below $0.01 \mathrm{~m} / \mathrm{s}$; i.e., the vehicles remain stationary during these finite time intervals, which means $T_{\mathrm{jam}}>0$. As a result, vehicles in such a mode will advance for a moment and stop for a moment. Such velocity oscillations are hence named as "stop and go" periodic 
oscillation. For $k=4,5, \min _{t \in[0,1000]} v_{1}(t)$ is very close but up to $0.01 \mathrm{~m} / \mathrm{s}$, and $\max _{t \in[0,1000]} v_{1}(t)$ is below but very close to the desired speed $v^{0}$.
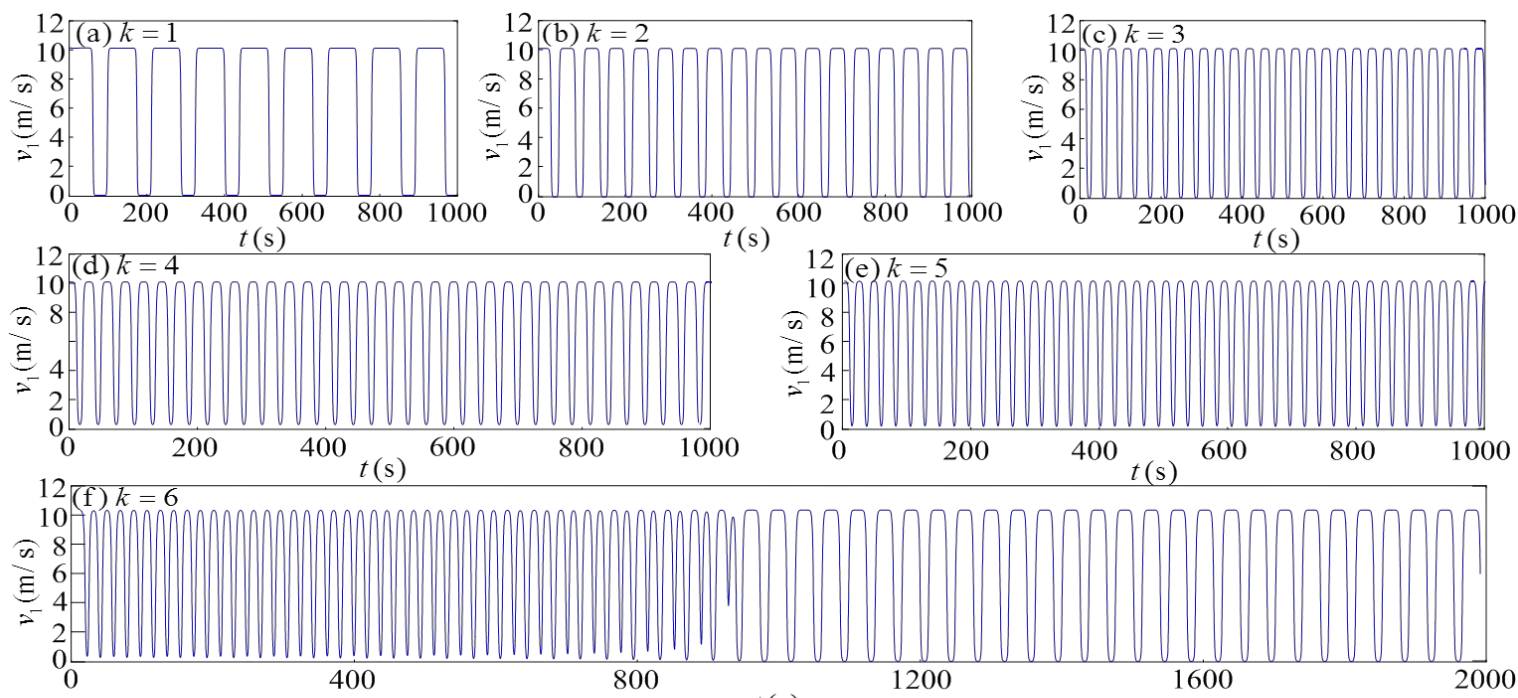

$t(\mathrm{~s})$

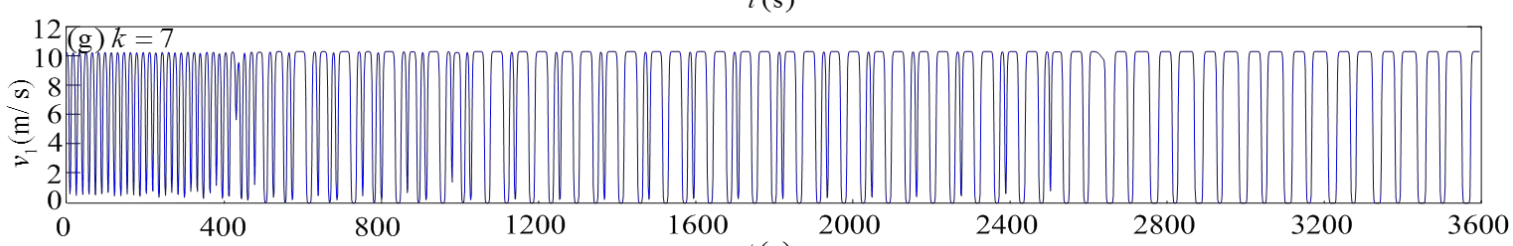

$t(\mathrm{~s})$

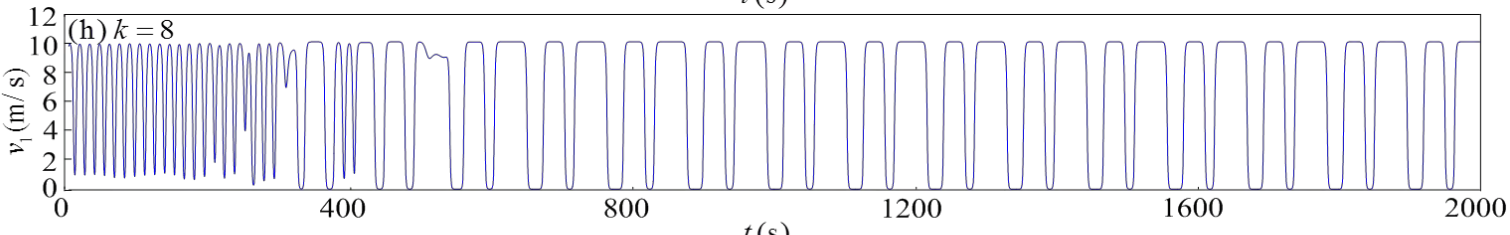

$t(\mathrm{~s})$
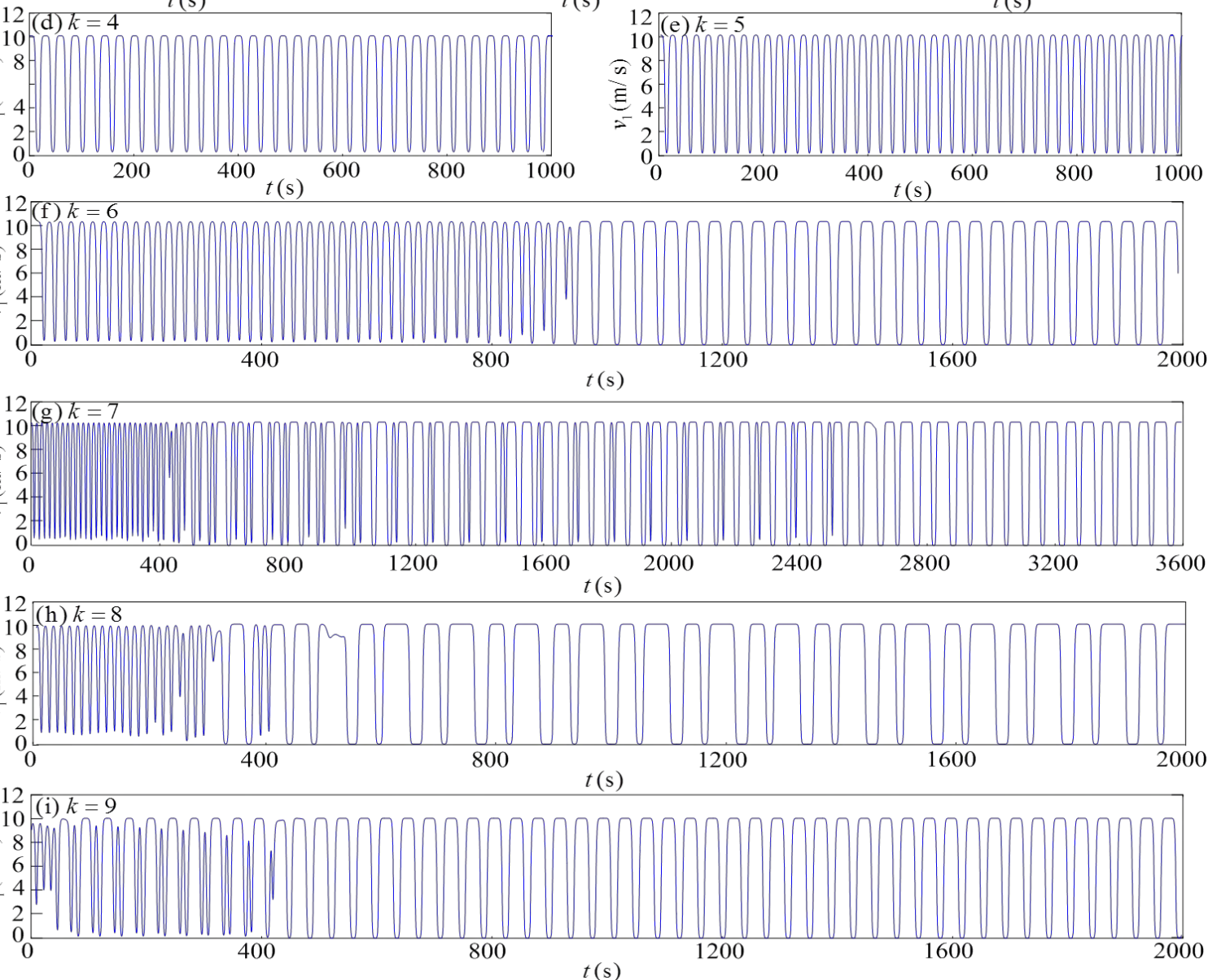

Figure 3: The time histories of the velocity of the first car for wave numbers $k=1, \ldots, 9$.

In these cases, vehicles will not stop and stay stationary $\left(T_{\mathrm{jam}}=0\right)$, but will move at speeds with wide fluctuations. Such velocity oscillations are named as "large amplitude" periodic oscillations. For $k=7,8$, similar "stop and go" phenomena are observed. Additionally, as stated in Section 4, the oscillations finally evolve into period-doubling oscillations. Therefore, they are named as "stop and go" period-doubling oscillations. Consequently, according to the characteristics of velocity distribution in Fig. 3 and based on our definition of stationary, three types of stable oscillation exist when $\tau=1$, namely, "stop and go" periodic oscillations for $k=1,2,3,6,9$, "large amplitude" periodic oscillations for $k=4,5$, and "stop and go" period-doubling oscillations for $k=7,8$.

In order to visualize traffic patterns corresponding to these three types of oscillation, we choose the wave numbers $k=1, k=5$ and $k=8$ as examples to illustrate the "stop and go" periodic oscillation, "large amplitude" periodic oscillation, and "stop and go" period-doubling oscillation respectively. With constant function along the interval $[-\tau, 0]$ as initial conditions, the spatio-temporal diagrams (i.e., position of each car at each time) for these three types of oscillations are shown in Figs. 4 a, b, and c respectively. 
Notice that in the spatio-temporal diagrams $4 \mathrm{a}$ and $4 \mathrm{c}$, curves have some horizontal sections. Such horizontal curves mean that the vehicles do not go forward for some time; i.e., they are jammed. Hence, the horizontal sections in the spatio-temporal diagram are named as congested cluster. Once vehicles have entered the congested clusters, they have to wait some time. As a result, congested cluster is an indicator of traffic jams.
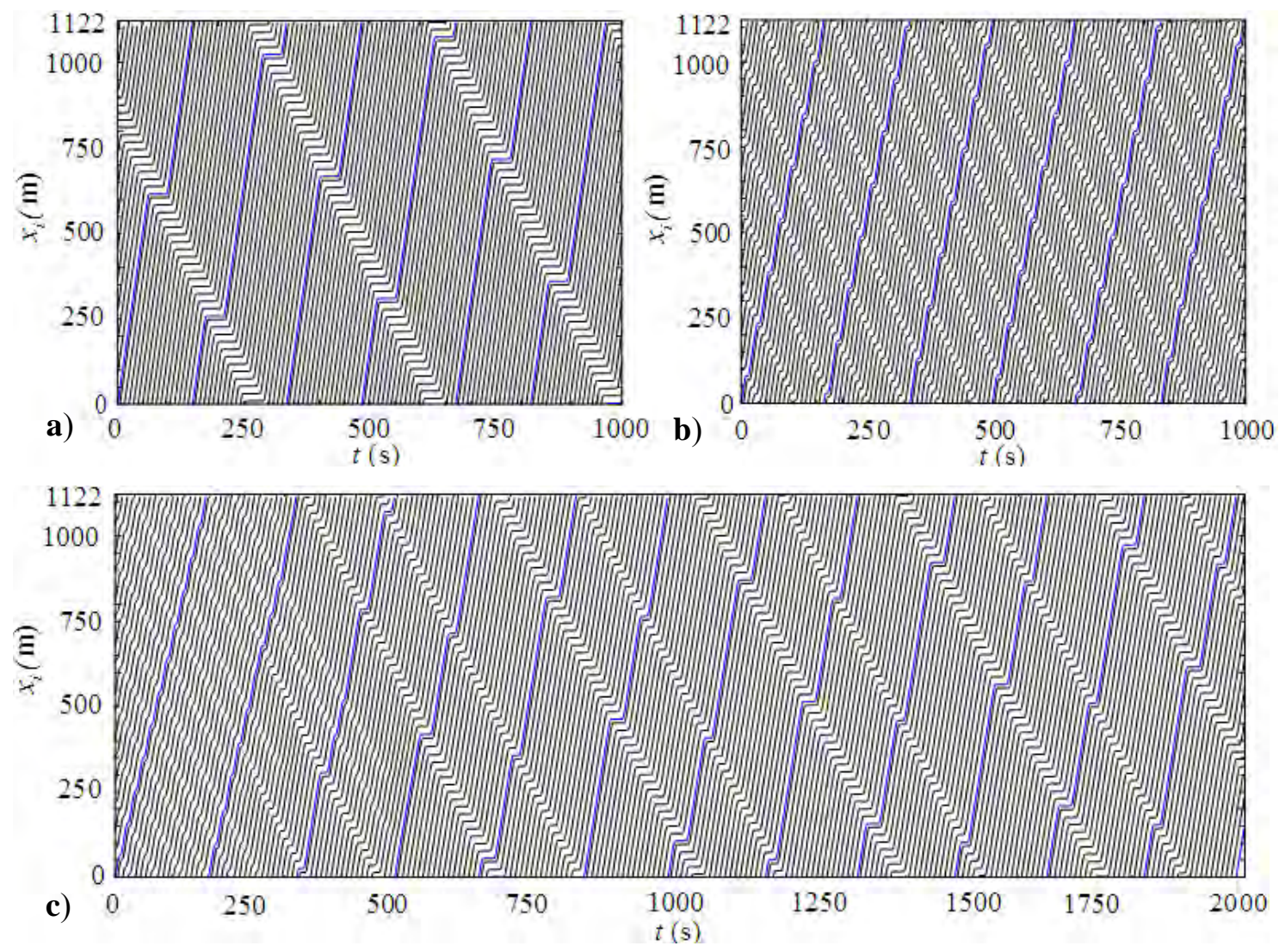

Figure 4: Spatiotemporal diagram for different wave numbers: a) $k=1$, b) $k=5$ and c) $k=8$; the trajectory of the first vehicle is emphasized as blue curves.

In Fig. $4 \mathrm{a}$, it is observed that each vehicle has horizontal sections in position, indicating that the vehicles will be jammed for some time. Moreover, it reads from Fig. 4 a that each vehicle wait exactly the same time at each congested cluster; i.e., $T_{\text {jam }}=33 \mathrm{~s}$ for $k=1$. Such a traffic pattern is hence referred to as "width-equal traffic jam". In this traffic pattern, drivers need to decrease or increase their velocities more than once during one circle, but have to face one or two traffic jams during one circle and wait the same time in every congested cluster once having entered.

In Fig. 4 b, one can see that there is no horizontal section in position for each vehicle; i.e., $T_{\mathrm{jam}}=0$ for $k=5$, which means that the velocities are always positive (vehicles without being completely stopped) along the ring for one circle. However, in such a case, drivers will frequently apply the brake to decelerate and then press the gas pedal to accelerate, seven or eight times during one circle. According to our definition, no traffic jam will occur even though the minimum velocity is very small (but NOT zero). Such traffic a pattern is referred to as "almost traffic jam".

In Fig. 4 c, after entering a steady state, each vehicle also has several horizontal segments in position, with total waiting time of $T_{\mathrm{jam}}=20 \mathrm{~s}$, but is discontinuously divided into a wide traffic jam with $T^{1}{ }_{\text {jam }}=15 \mathrm{~s}$ and a narrow jam with $T^{2}$ jam $=5 \mathrm{~s}$, as marked in Fig. $4 \mathrm{c}$. In this traffic pattern, once entering the congested clusters, vehicles will meet one after another traffic jam. This pattern is referred to as the "wide-alternated traffic jam". 
Table I: The classification of traffic jams.

\begin{tabular}{|c|c|c|c|c|c|}
\hline$k$ & transient oscillation & \multicolumn{2}{|c|}{ steady oscillation } & traffic pattern & $T_{\text {jam }}(\mathrm{s})$ \\
\cline { 1 - 2 } $1,2,3$ & stable & \multirow{2}{*}{ period } & stop and go & width-equal & \multirow{2}{*}{33} \\
\cline { 1 - 2 } 6,9 & unstable & period & large amplitude & almost traffic jam & 0 \\
\hline 4,5 & stable & period-doubling & stop and go & width-alternated & $T_{\text {jam }}^{1}=15, T_{\text {jam }}^{2}=5$ \\
\hline
\end{tabular}

Table I lists the characteristics of the three types of oscillation and their corresponding traffic patterns. These three different traffic patterns can coexist, and the initial conditions determine which one is approached.

\section{CONCLUSION}

In this paper, a full velocity difference model for traffic flow with drivers' reaction time delay is developed, which comprehensively includes the drivers' reaction with respect to the stimuli of headway, its own velocity and velocity difference. In order to study the effects of time delay on traffic dynamics, stability analysis and bifurcation analysis are carried out. The local stability of the uniform flow equilibrium is investigated when drivers' reaction time delay is varied, which reveals that the traffic system will undergo Hopf bifurcations at critical points and uniform flow becomes unstable, inducing oscillating solutions. After that, numerical continuation techniques are employed to investigate the bifurcation when reaction-time delay is far from the critical point. Following the branches of oscillations, fold, Neimark-Sacker and period-doubling bifurcations are detected, which cause qualitative changes of the oscillating solutions. It is shown that several stable and unstable oscillating solutions can coexist when time delay is larger than the critical value. Numerical simulations are performed to show that all periodic oscillations will eventually evolve into stable periodic or stable period-doubling oscillations. These two stable oscillations can be further classified into three types according to their velocity characteristic, which are "large amplitude" periodic oscillation, "stop and go" periodic oscillation and "stop and go" period-doubling oscillation. Finally, spatial patterns corresponding to these oscillations are shown in a spatio-temporal diagram. We show that multiple types of traffic jams, namely, "almost traffic jams", "width-equal traffic jams" and "width-alternated traffic jams", coexist.

The presented paper establishes a full velocity difference model with drivers' reaction time delay, the dynamics of which are studied in terms of bifurcation. The obtained results provide a classification of traffic jam, which significantly improves our understanding of the dynamics of traffic flow as well as the mechanism of traffic jams induced by driver's reaction time delay. In further work, more complicate oscillations and traffic patterns will be studied which have arisen near the bifurcating oscillations.

\section{REFERENCES}

[1] Helbing, D. (2001). Traffic and related self-driven many-particle systems, Reviews of Modern Physics, Vol. 73, No. 4, 1067-1141, doi:10.1103/RevModPhys.73.1067

[2] Lighthill, M. J.; Whitham, G. B. (1955). On kinematic waves. II. A theory of traffic flow on long crowded roads, Proceedings of the Royal Society A: Mathematical, Physical and Engineering Sciences, Vol. 229, No. 1178, 317-345, doi:10.1098/rspa.1955.0089

[3] Nagel, K.; Schreckenberg, M. (1992). A cellular automaton model for freeway traffic, Journal de Physique I, Vol. 2, No. 12, 2221-2229, doi:10.1051/jp1:1992277

[4] Bando, M.; Hasebe, K.; Nakayama, A.; Shibata, A.; Sugiyama, Y. (1995). Dynamical model of traffic congestion and numerical simulation, Physical Review E, Vol. 51, No. 2, 1035-1042, doi:10.1103/PhysRevE.51.1035 
[5] Treiber, M.; Kesting, A.; Helbing, D. (2006). Delays, inaccuracies and anticipation in microscopic traffic models, Physica A: Statistical Mechanics and its Applications, Vol. 360, No. 1, 71-88, doi:10.1016/j.physa.2005.05.001

[6] Laval, J. A.; Leclercq, L. (2010). A mechanism to describe the formation and propagation of stop-and-go waves in congested freeway traffic, Philosophical Transactions of the Royal Society A: Mathematical, Physical and Engineering Sciences, Vol. 368, No. 1928, 4519-4541, doi:10.1098/rsta.2010.0138

[7] Orosz, G.; Wilson, R. E.; Stépán, G. (2010). Traffic jams: dynamics and control, Philosophical Transactions of the Royal Society A: Mathematical, Physical and Engineering Sciences, Vol. 368, No. 1928, 4455-4479, doi:10.1098/rsta.2010.0205

[8] Igarashi, Y.; Itoh, K.; Nakanishi, K.; Ogura, K.; Yokokawa, K. (2001). Bifurcation phenomena in the optimal velocity model for traffic flow, Physical Review E, Vol. 64, No. 4, Paper 047102, doi:10.1103/PhysRevE.64.047102

[9] Doedel, E. J.; Champneys, A. R.; Fairgrieve, T. F.; Kuznetsov, Y. A.; Sandstede, B.; Wang, X. (1998). Auto97. Continuation and bifurcation software for ordinary differential equations, from ftp://ftp.cs.concordia.ca/pub/doedel/auto, accessed on 16-05-2014

[10] Gasser, I.; Sirito, G.; Werner, B. (2004). Bifurcation analysis of a class of 'car following' traffic models, Physica D: Nonlinear Phenomena, Vol. 197, No. 3-4, 222-241, doi:10.1016/j.physd. 2004.07.008

[11] Greenshields, B. D. (1936). Reaction time in automobile driving, Journal of Applied Psychology, Vol. 20, No. 3, 353-358

[12] Bando, M.; Hasebe, K.; Nakanishi, K.; Nakayama, A. (1998). Analysis of optimal velocity model with explicit delay, Physical Review E, Vol. 58, No. 5, 5429-5435, doi:10.1103/PhysRevE.58.5429

[13] Davis, L. C. (2003). Modifications of the optimal velocity traffic model to include delay due to driver reaction time, Physica A: Statistical Mechanics and its Applications, Vol. 319, 557-567, doi:10.1016/S0378-4371(02)01457-7

[14] Yu, L.; Li, T.; Shi, Z.-K. (2010). Density waves in a traffic flow model with reaction-time delay, Physica A: Statistical Mechanics and its Applications, Vol. 389, No. 13, 2607-2616, doi:10.1016/j.physa.2010.03.009

[15] Orosz, G.; Wilson, R. E.; Szalai, R.; Stépán, G. (2009). Exciting traffic jams: nonlinear phenomena behind traffic jam formation on highways, Physical Review E, Vol. 80, No. 4, Paper 046205, doi:10.1103/PhysRevE.80.046205

[16] Engelborghs, K.; Luzyanina, T.; Roose, D. (2002). Numerical bifurcation analysis of delay differential equations using DDE-BIFTOOL, ACM Transactions on Mathematical Software, Vol. 28, No. 1, 1-21

[17] Yu, L.; Shi, Z.-K.; Li, T. (2014). A new car-following model with two delays, Physics Letters A, Vol. 378, No. 4, 348-357, doi:10.1016/j.physleta.2013.11.030

[18] Wagner, P. (2010). Fluid-dynamical and microscopic description of traffic flow: a data-driven comparison, Philosophical Transactions of the Royal Society A: Mathematical, Physical and Engineering Sciences, Vol. 368, No. 1928, 4481-4495, doi:10.1098/rsta.2010.0122

[19] Shamoto, D.; Tomoeda, A.; Nishi, R.; Nishinari, K. (2011). Car-following model with relative-velocity effect and its experimental verification, Physical Review E, Vol. 83, No. 4, Paper 046105, doi:10.1103/PhysRevE.83.046105

[20] Zhou, J.; Shi, Z.-K.; Cao, J.-L. (2014). Nonlinear analysis of the optimal velocity difference model with reaction-time delay, Physica A: Statistical Mechanics and its Applications, Vol. 396, 77-87, doi:10.1016/j.physa.2013.11.007

[21] Jiang, R.; Wu, Q.; Zhu, Z. (2001). Full velocity difference model for a car-following theory, Physical Review E, Vol. 64, No. 1, Paper 017101, doi:10.1103/PhysRevE.64.017101

[22] Green, M. (2000). "How long does it take to stop?" Methodological analysis of driver perception-brake times, Transportation human factors, Vol. 2, No. 3, 195-216 